\title{
Análise do diagrama do modelo de promoção da saúde de Nola J. Pender
}

\author{
Analysis of diagram the health promotion model of Nola J. Pender \\ Análisis del diagrama modelo de la promoción de la salud de Nola J. Pender
}

\author{
Janaína Fonseca Victor ${ }^{1}$, Marcos Venícios de Oliveira Lopes², Lorena Barbosa Ximenes ${ }^{3}$
}

\begin{abstract}
RESUMO
Introdução: No Brasil, não encontramos referencias teóricas quanto ao uso do Modelo de Promoção da Saúde de Nola J. Pender , apesar de sua ampla utilização por pesquisadores americanos, asiáticos e europeus, para estudar comportamentos que levam à promoção da saúde. Objetivo: Analisar o diagrama do modelo de Promoção da saúde de Nola J. Pender. Método: Utilizou-se o método de análise de teorias proposto por Meleis em 1997. Resultados: A análise permitiu evidenciar que a representação gráfica do diagrama é de fácil compreensão, todos os componentes são descritos no texto pela autora, o que pode ser um fator facilitador para o uso deste modelo, por enfermeiros, na assistência e na pesquisa. No entanto, as ligações entre alguns componentes não são bem definidas. Evidenciou-se, ainda, que alguns termos do diagrama são de difícil tradução para o idioma português. Considerações finais: Ao analisar o diagrama do modelo citado acima, pudemos ampliar o leque de opções quanto aos referenciais teóricos que incrementam as ações de enfermagem.
\end{abstract}

Descritores: Promoção da saúde; Modelos de enfermagem

\begin{abstract}
Background: No theoretical references regarding the use of the Health Promotion Model, by Nola J. Pender, could be found in Brazil, in spite of its extensive use by American, Asiatic and European researchers to analyze behaviors leading to health promotion. Aim: To analyze the diagram of the Health Promotion Model, by Nola J. Pender. Method: To use the method to analyze theories proposed by Meleis 1997. Results: the analysis allowed to evidence that the graphical representation of the diagram is of easy understanding, all the components are described in the text by the author, which can be a facilitator factor to use this model by nurses in the assistance and research. However, the linkings between some components are not well defined. It was still demonstrated that some terms of the diagram are of difficult translation into the Portuguese language. Final Considerations: when analyzing the diagram of the model above mentioned we could increase our options regarding theoretical referentials, which enhanced the nursing actions.
\end{abstract}

Keywords: Health promotion; Models, nursing

\begin{abstract}
RESUMEN
Introducción: En Brasil, no encontramos referencias teóricas cuanto al uso del Modelo de la Salud, de Nola J. Pender, a pesar de su amplia utilización por pesquisadores americanos, asiáticos y europeos para estudiar los comportamientos que llevan a promoción de la salud. Objetivo: Analizar el diagrama del Modelo de Promoción de la Salud de Nola J. Pender. Método: Utilizou-se el método de análises de teorías presentado por Meleis en 1997. Resultados: el análisis ha permitido evidenciar que la representación gráfica del diagrama es de fácil comprensión, todos los componentes se describen en el texto por el autor, que puede ser un factor de facilitación para el uso de este modelo por las enfermeras en el cuidado y investigación. Sin embargo, las ligaciones entre algunos componentes no están bien definidas. Todavía, fue probado que algunos términos del diagrama son de difícil traducción para la lengua portuguesa. Consideraciones finales: al analizar el diagrama del modelo arriba mencionado, pudimos ampliar la gama de opciones cuanto a los refrenciales teóricos que incrementan las acciones de enfermería.
\end{abstract}

Descriptores: Promoción de la salud; Modelos de enfermería

\footnotetext{
1 Doutoranda em enfermagem pela Universidade Federa do Ceará - UFC - Fortaleza (CE), Brasil.

2 Professor Adjunto da Universidade Federa do Ceará - UFC - Fortaleza (CE), Brasil.

3 Professora Adjunto da Universidade Federa do Ceará - UFC - Fortaleza (CE), Brasil.
} 


\section{INTRODUÇÃOO}

Na literatura é crescente o número de trabalhos que procuram refletir sobre a aplicabilidade dos modelos e teorias de enfermagem; tal tendência possibilita validação e construção de novas formas de interpretar a enfermagem.

Mesmo com o avanço na ampliação de publicações que versam sobre modelos e teorias de enfermagem, observamos que são privilegiadas nesses estudos, a Teoria de Alcance de Metas de Imogenes King, a Teoria do Cuidado Cultural de Leininger, o Modelo de Adaptação de Callista Roy, a Teoria Humanista de Paterson e Zderard e a Teoria do Déficit do Autocuidado de Orem ${ }^{(1-7)}$.

Este fato pode ser explicado pela larga aplicação destas teorias na prática da enfermagem, o que torna imprescindível à continuidade de estudos dessa natureza. Portanto, a perspectiva de muitos trabalhos na área de enfermagem tem se voltado para as teorias de necessidades, teorias sistêmicas e humanísticas, visando estudos teóricos ou práticos da utilização desses modelos e teorias. Sabemos que estudos assim realizados favorecem a melhoria da qualidade do cuidado, o que contribui claramente para a promoção da saúde. No entanto, é oportuno nos aprofundarmos quanto à promoção da saúde, como um modelo-teórico para a prática de enfermagem.

Observamos que após discussões suscitadas nas conferências internacionais de promoção da saúde (Otawa, Adelaide, Sundsvall, Bogotá, Jacarta), as atividades de promoção da saúde passaram a ser amplamente estimuladas, sendo compreendidas como o estímulo à utilização das habilidades e conhecimentos das pessoas, a adoção de estilo de vida saudável, a mudança de comportamento, o lazer, e a prática de atividade física ${ }^{(8)}$.

Desde então, o termo promoção da saúde passou a ser utilizado por todos os profissionais de saúde, sendo crescente a participação dos enfermeiros em atividades que conduzem a promoção da saúde. Contudo, ainda são tímidas as propostas de estudos de eixos norteadores para a promoção da saúde, especialmente, na adoção de modelos e teorias que fundamentem essas experiências, conferindo um grau limitado no planejamento, execução e avaliação dessas ações ${ }^{(9-11)}$.

A utilização de modelos e teorias no campo da promoção da saúde pode facilitar na compreensão dos determinantes dos problemas de saúde, orientar nas soluções que respondem às necessidades e interesses das pessoas envolvidas. Além disso podem contribuir para a promoção de conhecimento, reflexão e decisão no ato de cuidar e agir, possibilitando maiores chances no alcance dos objetivos propostos, tanto para a promoção da saúde, quanto para a prevenção de doenças ${ }^{(12-13)}$.

Entendemos modelos, como referenciais conceituais, representações construídas de algum aspecto do ambi- ente, utilizando as abstrações como blocos embasadores. O modelo permite construir uma base mais sólida, para uma prática mais informada ${ }^{(14)}$.

Dentre os modelos e teorias de enfermagem que possam ser aplicados para desenvolver cuidados de enfermagem no âmbito da promoção da saúde, podemos citar o Modelo de Promoção da Saúde de Pender ${ }^{(14)}$. Este fornece uma estrutura simples e clara, em que o enfermeiro pode realizar um cuidado de forma individual, ou reunindo as pessoas em grupo, permitindo planejamento, intervenção e avaliação de suas ações. Este modelo vem sendo amplamente utilizado por muitos pesquisadores americanos, asiáticos e europeus, para estudar comportamentos que levam à promoção da saúde ${ }^{(15-17)}$.

No entanto, no Brasil, não encontramos referencias teóricas quanto ao uso deste Modelo na prática da enfermagem. E acreditamos que modelos não são verdades absolutas, mas caminhos a percorrer, e que estudos analíticos incrementam a pesquisa, facilitam escolhas, além de revelarem possibilidades e limitações que podem enriquecer a prática profissional, por isso, realizamos o presente estudo com o objetivo de analisar o diagrama do Modelo de Promoção da Saúde de Nola J. Pender, utilizando o método de análise proposto por Meleis em seu livro Nursing theory: a elusive mirage or a mirror of reality ${ }^{(18)}$.

\section{MÉTODO}

Trata-se de um estudo de análise, proposto Meleis ${ }^{(18)}$, do diagrama do Modelo de Promoção da Saúde de Nola J. Pender ${ }^{(19)}$.

O modelo engloba: descrição, análise, crítica, teste e apoio. A descrição pode ser feita através de seus componentes estruturais e funcionais. A análise é definida como um processo de identificação de partes e componentes. A crítica é um exame ou estimativa de uma situação. O teste teórico refere-se a uma avaliação para a utilidade da teoria. Já o teste de apoio, verifica alternativas de validação, em congruência com a natureza da disciplina $^{(18)}$.

Quanto aos aspectos propostos por Meleis ${ }^{(18)} \mathrm{em} \mathrm{seu}$ modelo, utilizamos, particularmente neste estudo, a crítica da teoria que objetiva estabelecer uma relação entre estrutura e função, sendo constituída de oito componentes (clareza, consistência, simplicidade e complexidade, tautologia e teleologia, diagrama, circulo de contágio, utilidade e componentes externos). Para a análise do Modelo de Promoção da Saúde de Nola J. Pender optamos, dentre os componentes da crítica da teoria acima citados, o diagrama.

A ênfase em estudar o diagrama ocorreu por ser este, a melhor forma para se aproximar do modelo ${ }^{(18)}$. Analisamos, em particular, a apresentação gráfica e visual, 
(o desenho do modelo representado por ligações, setas, retângulos) a representação lógica (a seqüência das ligações e os tipo de interações) e a clareza (definições operacionais). Estes três elementos, quando utilizados de forma satisfatória, facilitam a compreensão do modelo ou teoria. Para tal, a análise foi norteada pelas seguintes questões propostas por Meleis ${ }^{(18)}$ : inclui grandes conceitos? A apresentação gráfica realçou a compreensão dos diferentes componentes da teoria, é uma representação real do texto? O diagrama é claro e bem definido? As direções de ligações são indicadas?

A partir desses questionamentos propostos ${ }^{(18)}$, analisamos o diagrama do Modelo de Promoção da Saúde de Pender ${ }^{(19)}$, dando possibilidades quanto a uma nova perspectiva para enfermagem brasileira na promoção da saúde.

\section{APRESENTAÇÃO DO MODELO DE PROMOÇÃO DA SAÚDE}

Para melhor compreensão do diagrama, apresentamos, inicialmente, alguns aspectos pertinentes do modelo. O Modelo Teórico de Promoção da Saúde de Pender, desenvolvido na década de 80 por Nola J. Pender, professora emérita da Escola de Enfermagem da Universidade de Michigan - Estados Unidos, repousa sobre a concepção de promoção da saúde, definida como aquelas atividades voltadas para o desenvolvimento de recursos que mantenham ou intensifiquem o bem-estar da pessoa. $\mathrm{O}$ modelo surge como uma proposta para integrar a enfermagem à ciência do comportamento, identificando os fatores que influenciam comportamentos saudáveis, além de ser um guia para explorar o complexo processo biopsicossocial que motiva indivíduos para se engajarem em comportamentos produtores de saúde ${ }^{(19)}$.

O Modelo de Promoção da Saúde é fundamentalmente, um modelo de enfermagem, podendo ser usado para implementar e avaliar ações de promoção da saúde, permitindo avaliar o comportamento que leva à promoção da saúde, pelo estudo da inter-relação de três pontos principais: 1 . as características e experiências individuais, 2. os sentimentos e conhecimentos sobre o comportamento que se quer alcançar e 3. o comportamento de promoção da saúde desejável (ver figura1). Na construção do Modelo de promoção da saúde, a autora utilizou, como bases teóricas a Teoria da Aprendizagem Social e o Modelo de Avaliar Expectativas, ambos da psicologia ${ }^{(19)}$.

\section{Características}

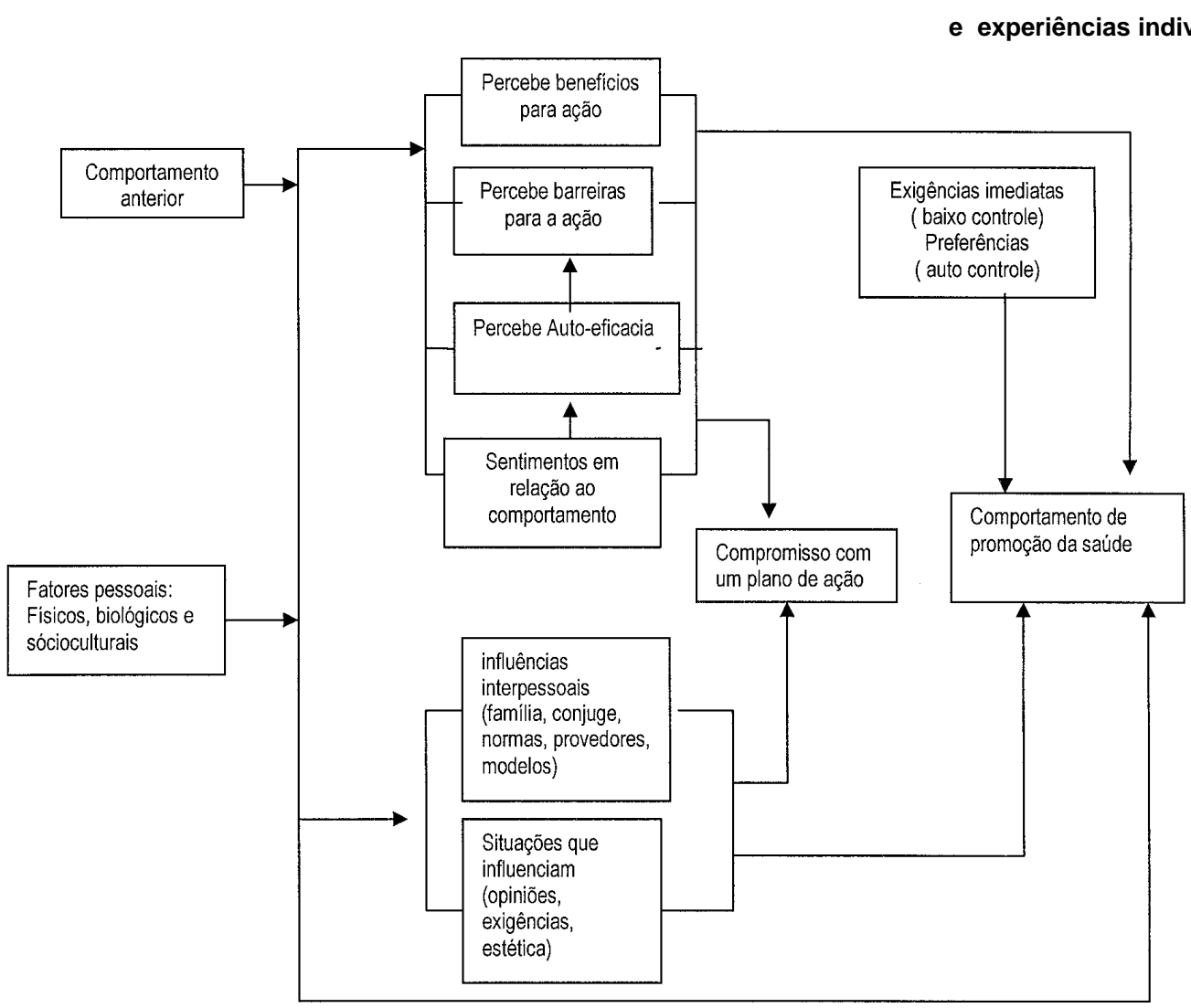

3. Resultado do Comportamento e experiências individuais

Figura 1 - Diagrama do Modelo de Promoção a Saúde. Traduzido de Health Promotion in Nursing Practice ${ }^{(19)}$ 
ANÁLISE DO DIAGRAMA DO MODELO TEÓRICO DE PROMOÇÃO DA SAÚDE DE NOLA J. PENDER

Para analisar o diagrama do Modelo de Promoção da Saúde de Nola J. Pender optamos em utilizar as perguntas propostas por Meleis ${ }^{(18)}$ em seu modelo de análise da crítica da teoria, ora citadas na metodologia.

Inclui grandes conceitos?

Grandes conceitos, referem-se aos elementos que constituem um modelo ou teoria de enfermagem, conceitos de ambiente, de saúde, de enfermagem e de pessoa $^{(18)}$. Neste Modelo, estes conceitos aparecem diluídos em todo o livro, não especificamente no capítulo que enfatiza o modelo em si.

O termo saúde é visto sob os aspectos individual, familiar e comunitário, com ênfase na melhoria do bemestar, no desenvolvimento de capacidades e não como ausência de doenças, devendo ser estudado durante todo o processo de desenvolvimento do ser humano, levando em consideração idade, raça e cultura, numa perspectiva holística $^{(19)}$.

Quanto ao ambiente, este deve ser compreendido como resultado das relações entre indivíduo e seu acesso a recursos de saúde, sociais e econômicos, relatando que esta relação proporciona um ambiente saudável. O conceito de pessoa é definido como aquele capaz de tomar decisões, resolver problemas; a ênfase, contudo, está no seu potencial de mudar comportamentos de saúde ${ }^{(19)}$.

O conceito enfermagem surge no texto, intimamente relacionado às intervenções e estratégias que a enfermeira deve dispor para o comportamento de promoção da saúde, sendo que um dos principais papeis do enfermeiro é o estímulo ao autocuidado ${ }^{(19)}$.

Apesar desta construção feita por Pender ${ }^{(19)}$, abordando estes conceitos no seu Modelo, estes não estão explícitos no diagrama. Contudo, entendemos que as variáveis representadas no diagrama resgatam tais conceitos de forma subjetiva. Ë necessário que os que desejam utilizar o Modelo de Promoção da Saúde de Pender aprofundem sua leitura para se apropriar de tais conceitos, pois o diagrama, por si, não possibilita essa compreensão. De acordo com Meleis ${ }^{(18)}$, estes conceitos são elementos-chave para viabilizar a aplicação do Modelo, na prática.

A apresentação gráfica realçou a compreensão dos diferentes componentes da teoria, é uma representação real do texto?

O Modelo de Promoção da saúde é composto por três grandes componentes, que estão representados graficamente no diagrama. Os componentes estão subdivididos em variáveis, que apresentam-se da seguinte for- ma: 1. características e experiências individuais, que compreende o comportamento anterior, comportamento que deve ser mudado e os fatores pessoais, que são divididos em fatores biológicos( idade, índice de massa corpórea, agilidade); psicológicos ( auto-estima, automotivação) e sócioculturais (educação, nível sócioeconômico).

O componente 2. sentimentos e conhecimentos sobre o comportamento que se quer alcançar, é o núcleo central do diagrama, sendo formado pelas variáveis; percebe benefícios para ação, representações mentais positivas, que reforçam as consequiências de adotar um comportamento; percebe barreiras para ação, percepções negativas sobre um comportamento, são vistas como dificuldades e custos pessoais; percebe auto-eficácia, julgamento das capacidades pessoais de organizar e executar ações; sentimentos em relação ao comportamento, reflete uma reação emocional direta ou uma resposta nivelada ao pensamento que pode ser positivo, negativo, agradável ou desagradável; $\underline{\text { in- }}$ fluências interpessoais, o comportamento pode ou não ser influenciado por outras pessoas, família, cônjuge, provedores de saúde, ou por normas e modelos sociais; influências situacionais, o ambiente pode facilitar ou impedir determinados comportamentos de saúde (Figura 1).

O componente 3. resultado do comportamento abrange o compromisso com o plano de ação, ações que possibilitem o indivíduo a manter-se no comportamento de promoção da saúde esperado, isto é, as intervenções de enfermagem ; exigências imediatas e preferências, as pessoas têm baixo controle sobre os comportamentos que requerem mudanças imediatas, enquanto que as preferências pessoais exercem um alto controle sob as ações de mudança de comportamento; comportamento de promoção da saúde, resultado da implementação do Modelo de Promoção da Saúde (Figura 1).

Todos os componentes e variáveis do modelo apresentados no texto original, aparecem no diagrama, sendo uma representação real do conteúdo apresentado; no entanto, quanto à descrição das variáveis, essa representação é limitada, pois o diagrama possui 11 variáveis; contudo, somente as variáveis influências interpessoais e influências situacionais são descritas no diagrama (Figura 1).

As demais variáveis aparecem no diagrama como tópicos sem definições do que representam. Esta apresentação, pode influenciar na utilização do Modelo, tanto no aspecto de limitação como de erro de interpretação, especialmente, por serem descritas em outro idioma, algumas variáveis podem não ser compreendidas em seu sentido real ${ }^{(18)}$. 
O diagrama é claro e bem definido, as direções de ligações são indicadas?

Quanto ao aspecto das direções de ligações do diagrama, estas estão indicadas por linhas contínuas e setas que unem retângulos; cada variável está ligada a uma ou mais variáveis, o que gera uma representação gráfica aparentemente clara (ver figura 1). No entanto, essa clareza é limitada, quanto à compreensão das variáveis. $\mathrm{O}$ pesquisador necessita buscar na descrição do texto original o que algumas variáveis representam, para então, utilizar o modelo.

Ao analisarmos a variável comportamento anterior representado no diagrama, a direção da seta indica que este interfere diretamente no comportamento de promoção da saúde, como também, nos demais componentes, isto é ratificado pela própria autora quando refere que esta variável é o ponto de partida do modelo, isto é, a condição padrão para a elaboração das intervenções de enfermagem, além de influenciar diretamente no comportamento de promoção da saúde.

A representação gráfica dos fatores pessoais (biológicos, psicológico e sócioculturais), indica que todos estes interferem diretamente no comportamento de promoção da saúde (ver figura 1). Entretanto, Pender descreve no texto original que alguns fatores pessoais não podem ser incorporados como variáveis, para serem modificadas no comportamento, como exemplo, a raça, esta limitação não é clara no diagrama, nesta variável, a seta poderia ser tracejada, já que apresenta limitação.

Um dos aspectos importantes que Meleis ${ }^{(18)}$ ressalta é a análise das setas da representação gráfica das variáveis com relação ao texto original do modelo ou teoria. Por isso, decidimos fazer uma breve análise quanto à seqüência do texto original e o desenho do diagrama, apesar deste não ser o objetivo principal do estudo. Contudo, consideramos que esta etapa permitirá uma aproximação do leitor com o texto original, apesar de não termos condições de disponibilizá-lo, pela magnitude do conteúdo teórico e escrito.

As setas da representação gráfica das variáveis não são representadas satisfatoriamente, quanto à sequiência no texto e o desenho do diagrama. Segundo o diagrama, no componente 2 . comportamento específico, o primeiro tópico a ser discutido seria sentimentos relacionados ao comportamento, já que a seta parte desta variável; entretanto, no texto, a autora inicia a discussão com o último tópico percebe benefícios para a ação. Neste componente, as setas entre os retângulos dão uma idéia de continuidade e interrelação; contudo, não existe seta ligando percebe barreira para a ação e percebe beneficios para a ação, o que no texto traz claro que estes são complementares, visto que, Pender refere que a relação entre o número de benefícios e o número de barreiras poderá influenciar na mudança de comportamento (Figura 1).
Em relação às variáveis influências interpessoais $\mathrm{e}$ influências situacionais, estas, segundo a representação gráfica, interferem tanto no compromisso com o plano de ação como no comportamento de promoção da saúde, retratando fielmente o texto. Segundo Pender estas influências são fortemente evidenciadas em adolescentes que tendem a repetir comportamentos de outros. A autora exemplifica, ainda, que em lugares que possuem regras para não fumar, desenvolvem-se influências positivas nas pessoas.

O compromisso com o plano de ação segundo o diagrama é resultado de todos os demais componentes do Modelo; entretanto, a variável percebe barreiras para ação foi descrita no texto sob o aspecto do seu significado semântico, sem clara correlação com o plano de ação.

Outro ponto relevante é a ênfase que a autora dá no texto às variáveis exigências e preferências, como importantes no compromisso com o plano de ação, no entanto, esta relação não está representada no diagrama por nenhuma ligação. A representação gráfica do diagrama relaciona esta variável diretamente no comportamento de promoção da saúde.

O diagrama do Modelo de Promoção da Saúde de Pender ${ }^{(19)}$ é claro visualmente, e apresenta todos os componentes e variáveis descritos no texto. A relativa simplicidade da representação gráfica possibilita uma aproximação favorável à utilização do Modelo, visto que, segundo Meleis ${ }^{(18)}$, a compreensão do diagrama é passo inicial no entendimento de modelos e teorias.

\section{CONSIDERAÇÕES FINAIS}

A partir da análise do diagrama do Modelo de Promoção da Saúde de acordo com a análise da crítica da teoria ou modelo proposto por Meleis ${ }^{(18)}$, podemos visualizar que apesar de recente na enfermagem, o Modelo pode ser uma proposta de realizar intervenções de enfermagem no âmbito da promoção da saúde, em relação à mudança de comportamento, em bases mais sólidas.

Além de ser um Modelo novo criado em 1984, no Brasil não existem referências na literatura de enfermagem, o que nos mostra a importância de desenvolvermos estudos desta natureza, pois apesar do Modelo ter sido desenvolvido em outro país, este poderá ser adaptado para nossa realidade.

É oportuno ressaltar que ocorreram limitações, especialmente na tradução de alguns componentes e variáveis do modelo, que merecem ser aprofundados em estudos posteriores, pois, alguns termos não possuem clara correlação com o idioma português, o que nos fez refletir sobre possíveis erros que podem surgir na utilização do modelo, se este não for estudado com maior profundidade. 
Consideramos, ainda, que ao analisar o diagrama do Modelo de Promoção da Saúde de Nola J. Pender ${ }^{(19)}$ contribuímos para ampliar o leque de opções quanto aos referenciais teóricos que incrementam as ações de enfermagem, pois, estudos de análise de modelos ou teorias viabilizam uma aproximação do enfermeiro com os construtos teóricos, facilitando escolhas e adequações destes para sua prática profissional.

\section{REFERÊNCIAS}

1. Ximenes LB, Souza LJ, Pagliuca LMF. Teoria do cuidado cultural à luz de Barbara Barnun. Cogitare Enferm. 1999;14(1): 15-20.

2. Landim FLP, Frota MA, Pagliuca LMF. Teorias de enfermagem: reflexão como instrumento e construção da crítica ao conhecimento elaborado. Cogitare Enferm. 2001;6(1):50-6.

3. Moreira TMM, Araújo TL, Pagliuca LMF. Alcance da teoria de king junto a família de pessoas portadoras de hipertensão arterial sistêmica. Rev Gaúcha Enferm. 2001;22(1):74-89.

4. Dahmer AM, Larossa LL, Alves MCG, Thoferhn MB, Lange C. Humanização do cuidado de enfermagem aos clienetes em pós-operatório de cirurgia cardíaca. In: Santana MGS, Thoferhn MB, organizadores. Resignificando a teria e prática. Porto Alegre: Artes Médicas; 2001.

5. Bielemanm VM, Oliveira CCA.A enfermeira atuando frente ao grupo de clientes hipertensos. In: Santana MGS, Thoferhn MB, organizadores. Resignificando a teria e prática. Porto Alegre: Artes Médicas; 2001.

6. Lopes ND, Pagliuca LMF. Abordagem holística o termo pessoa em um estudo empírico: uma análise crítica. Rev Lat Am Enferm. 2002;10(6):825-30.

7. Fialho AVM, Pagliuca LMF, Soares E. Adequação da teoria do déficit de autocuidado no cuidado domiciliar à luz do modelo de barnum. Rev Lat Am Enferm. 1999; 10(5):715-20.

8. Fonseca LE. Promoção da saúde: carta de Otawa, declaração de Adelaide, Sundsvall e Santa Fé de Bogotá. Brasília: Ministério da Saúde; 2000.
9. Meles AM, Zago MMF. Análise da educação de clientes e pacientes na literatura brasileira de enfermagem. Rev Lat Am Enferm. 1999;7(5):85-94.

10. Kohlrausch E, Rosa NG. Relacionando os modelos assistenciais e as tendências pedagógicas em saúde: subsídios para a ação educativa da enfermeira. Rev Gaúcha Enferm. 1999; 20 (n.esp):113-22.

11. Victor JF. Educação em saúde na unidade básica de saúde da família: atuaçao do enfermeiro um estudo de caso. FortalezaCeará [tese]. Fortaleza:Universidade Federal do Ceará; 2002.

12. Naidoo J, Wills J. Health promotion: foundations for practice. London: Royal College of Nursing; 1994.

13. Freudenberg NE, Flay B, Parcel G, Rogers T, Walltersin NS. Individual and community capacity to prevent disease and promote health: searching of relevant theories and principles. Health Educ Q. 1996;26(3):290-306.

14. Polit DF, Hungle BP. Fundamentos de pesquisa em enfermagem. 3a ed. Porto Alegre: Artes Médicas; 1995.

15. Warren MT. The relationships of self-motivation and perceived personal competence to ngaging in a health-promotion lifestyle for men in rehabiblittion programs. [dissertation]. New York: New York University; 1993.

16. StuttsWC. Use of the Health Promotion Model to predict physical activity in adults. [dissertation]. Chapel Hill: University of North Carolina; 1997.

17. Pender NJ, Yang KP. Promotion phisical activity J Nurs Res 2002;10(1):57-64.

18. Meleis AI. Nursing theory: a elusive mirage or a mirror of reality. 3rd ed. Philadelphia: Lippincott; 1997.

19. Pender NJ, Murdaugh CL, Parsons MA. Health promotion in nursing practice. 4th ed. Englewood Cliffs: Prentice Hall; 2002. 